Revista Energia na Agricultura

ISSN 1808-8759

\title{
EFICÁCIA DO FLUTRIAFOL E DO FLUTRIAFOL + TIOFANATO METÍLICO APLICADOS COM GOTAS FINAS OU MÉDIAS NO CONTROLE DA FERRUGEM ASIÁTICA DA SOJA ${ }^{1}$
} MARIA APARECIDA PERES DE OLIVEIRA ${ }^{2} \&$ ULISSES ROCHA ANTUNIASSI $^{3}$

RESUMO: O presente trabalho teve por objetivo avaliar a influência da tecnologia de aplicação com o uso de gotas finas e médias no efeito residual dos fungicidas flutriafol e tiofanato metílico + flutriafol para o controle da ferrugem da soja (Phakopsora pachyrhizi Sydow \& Sydow). O delineamento experimental adotado foi em esquema fatorial 2 x 2 com 6 repetições, resultando em 4 tratamentos nas seguintes interações: 2 classes de gotas (finas e médias) e 2 fungicidas. A área experimental constituiu-se de 24 parcelas de aplicação $(50 \mathrm{~m}$ x $21 \mathrm{~m})$. Dentro de cada parcela foi posicionada uma área de avaliação central com 10 x $20 \mathrm{~m}$. Para cada parcela de avaliação havia uma testemunha não tratada fora da área aplicada (com as mesmas dimensões), localizada em direção oposta ao deslocamento do vento no momento da aplicação. As avaliações da eficiência dos tratamentos no controle da doença foram feitas mediante cálculo da incidência (porcentagem de plantas com ferrugem), severidade (intensidade ou níveis de infecção), desfolha (porcentagem de queda de folhas) e produtividade (pesagem das sementes das parcelas). Para cada uma das análises, as médias dos resultados foram comparados pelo cálculo do intervalo de confiança à $90 \%$. Com base nos dados de cada repetição e de suas respectivas testemunhas, foram calculados os percentuais de redução da severidade da ferrugem. O ensaio foi realizado em condições de controle curativo sendo que a ferrugem estava presente na área com média de $68,6 \%$ de infestação, com severidade média de 35,9\% no terço inferior e 4,57\% no terço superior. Os resultados de severidade, desfolha e produtividade mostraram que não houve diferenças estatísticas significativas entre os tratamentos. Entretanto, observou-se de maneira geral que em todos os dados houve tendência de melhores resultados para as aplicações com flutriafol, em comparação às aplicações com flutriafol + tiofanato metílico. Neste sentido, existe diferença no que se refere à dose proposta do princípio ativo flutriafol entre os tratamentos $\left(62,5 \mathrm{~g} \mathrm{ha}^{-1}\right.$ para o flutriafol e $60 \mathrm{~g} \mathrm{ha}^{-1}$ para o tiofanato metílico + flutriafol), mas a mesma é pequena. Não foram observadas diferenças significativas entre as aplicações com gotas finas e médias. Este fato pode ser explicado tanto pelas características de ação sistêmica do flutriafol como pelo tipo de controle realizado (curativo). O flutriafol, sendo um fungicida sistêmico, apresenta redistribuição dentro de cada folha, reduzindo o efeito de melhor cobertura gerado pelas gotas mais finas. No que se refere ao controle curativo, a quantidade de produto

\footnotetext{
${ }^{1}$ Parte da dissertação de mestrado do $1^{\mathrm{o}}$ autor

${ }^{2}$ Aluna do Curso de PG Energia na Agricultura - FCA/UNESP - Botucatu/SP - Brasil

${ }^{3}$ Orientador e Docente do Departamento de Engenharia Rural - FCA/UNESP - Botucatu/SP - Brasil
} 
depositado pode se tornar mais importante do que a cobertura, principalmente nas folhas da parte superior das plantas. Estas folhas estão mais sadias do que as inferiores, que geralmente são as primeiras a ficarem totalmente comprometidas, perdendo assim a importância na manutenção do desenvolvimento da planta. Por este motivo, as gotas médias acabam oferecendo um desempenho semelhante, apesar de não oferecer vantagens na cobertura das folhas da parte inferior das plantas. Este fato se torna importante, pois, na prática, as gotas médias estendem o período de trabalho na propriedade, visto que as gotas finas e muito finas têm muitas limitações, dentre elas, problemas de deriva e evaporação.

Palavras-chave: Pulverização, fungicida, pontas, gotas, tecnologia de aplicação.

\section{EFFICACY OF FLUTRIAFOL AND FLUTRIAFOL + THIOPHANATE METHYL SPRAYED WITH FINE OR MEDIUM DROPLETS FOR THE CONTROL OF ASIAN SOYBEAN RUST}

SUMMARY: The aim of this study was to evaluate the influence of fine and medium droplets in the performance of flutriafol and thiophanate methyl + flutriafol for the control of Asian Soybean Rust (Phakopsora pachyrhizi Sydow \& Sydow). The experiment was a 2 x 2 factorial design (2 droplet sizes $x 2$ fungicides) resulting in four treatments with six replications. The experimental area was set up with 24 plots (50 $\times 21 \mathrm{~m}$ ). In each plot there was a central assessment area with $10 \times 20 \mathrm{~m}$. To the each plot there was a non-treated area placed in opposed direction to the wind. The evaluations of rust control were made by of the calculation of incidence (percentage of plants with rust), severity (level of infection), defoliations and soybean yield. The results were analyzed by the calculation of the confidence interval at $90 \%$. The study was set up in curative control conditions with average infestation of 68.6\%, average severity in the lower part of the canopy was of $35.9 \%$ and on the upper parte it was $4.57 \%$. The results of severity, defoliation and productivity did not show statistical difference among the treatments. However, it was observed that in general there was tendency of better results with the application of flutriafol alone comparing with thiophanate methyl + flutriafol, since there was a small difference between two commercial products in the flutriafol active ingredient content ( $62.5 \mathrm{~g} \mathrm{ha}^{-1}$ to the flutriafol alone and $60 \mathrm{~g} \mathrm{ha}^{-1}$ to the thiophanate methyl + flutriafol). There was no statistical difference between fine and medium droplets. This fact can be explained by the characteristics of systemic action of the flutriafol and by the type of control made (curative). The flutriafol, being a systemic fungicide is less sensitive to the better coverage provided by the smaller droplets and, on the curative control, the amount of product deposited can became more import than coverage, mainly on the superior part of the leaves. These leaves are healthier than the inferior leaves that in general are the first to become completely compromised by the rust, losing importance to 
the plant. By this reason, medium droplets appear to have offered similar performance even not offering advantages in the leaf coverage. This fact may become important because in the practical side of the use of medium droplets, since this may increase the amount of time to spray, mainly because the fine and very fine droplets have more limitations related to drift and evaporation.

Keywords: Spray, fungicide, nozzle, droplets, application technology.

\section{INTRODUÇÃO}

A soja, no contexto das grandes culturas produtoras de grãos, foi a que mais cresceu em termos percentuais nos últimos 32 anos, tanto no Brasil quanto em nível mundial. De 1970 a 2003, o crescimento da produção global foi da ordem de $333 \%$ (de 43,7 para 189,2 milhões toneladas), enquanto culturas como trigo, arroz, milho, feijão, cevada e girassol cresceram, respectivamente $79 \%, 86 \%, 140 \%, 52 \%, 19 \%$, e $177 \%$ (EMBRAPA, 2004).

Como toda cultura exótica, a soja começou com excelente sanidade, porém, com poucos anos de cultivo comercial, as doenças começaram a aparecer, passando a representar um dos principais fatores limitantes ao aumento e à estabilidade do rendimento (YORINORI, 2002). As perdas anuais de produção por doenças de soja são estimadas em cerca de $15 \%$ a $20 \%$, entretanto, algumas doenças podem ocasionar perdas de quase 100\% (NUNES JÚNIOR, 2004).

A ferrugem da soja pode ser causada por duas espécies do gênero Phakopsora. Phakopsora meibomiae Arthur (Arthur) e Phakopsora pachyrhizi H. Sydow \& Sydow pertencentes à ordem Uredinales e classe Basidiomicota. As espécies são diferenciadas por análise de DNA e morfologia dos teliósporos e télias (BEDENDO, 1995; ONO et al., 1992).

Segundo Yorinori e Paiva (2002), após a primeira constatação de ferrugem Asiática (P. pachyrhizi ) no Paraguai e posteriormente no Brasil (PR) em 2001, a doença espalhou-se rapidamente por todo o Brasil, Paraguai, Bolívia e partes da Argentina.

A ferrugem é atualmente a doença mais importante na cultura da soja, principalmente devido à antecipação da desfolha e conseqüente deficiência na granação. Em algumas regiões do Brasil, nas safras $2002 / 03$ e 2004/05, as perdas chegaram a quase $100 \%$ devido à agressividade do patógeno, erro no momento de aplicação e deficiência nas pulverizações (CAMARGO, 2005). Quando a doença atinge a soja na fase de formação das vagens ou no início de granação, pode causar aborto e queda dessas vagens. Na Austrália e na Índia, respectivamente, perdas de até 80 e 90\% de produção foram registradas por Ono et al. (1992). 
No atual sistema de produção de culturas agrícolas, o uso de agrotóxicos é um dos principais pontos críticos, que, dentre outros fatores, elevam o custo de produção. De acordo com ANTUNIASSI (2004), após o aparecimento da ferrugem houve uma grande valorização da eficiência e da economia na tecnologia de aplicação de defensivos.

O controle químico com fungicidas, por ser o melhor método de controle da ferrugem, possui um custo que às vezes pode ser elevado. Esse custo é constituído de duas partes: custo da pulverização (custo operacional) e custo do fungicida. Segundo Melo Filho e Richetti (2004), o custo máquina por hectare de uma pulverização foi estimado em $\mathrm{R} \$ 7,78$. Quanto aos fungicidas, variaram de $\mathrm{R} \$ 46,00$ a $\mathrm{R} \$ 72,15$ por hectare. Assim, no caso de apenas uma aplicação de fungicida, a participação do custo do controle da ferrugem no custo de produção varia de $3,37 \%$ a $4,92 \%$ e o custo do tratamento corresponde ao valor de 1,70 a 2,50 sacos ha $^{-1}$ de soja.

Os fungicidas pertencentes ao grupo dos triazóis apresentam a característica de penetração e translocação na planta, mas sua ação sistêmica está, em $99 \%$ dos casos, condicionada ao movimento via xilema (AZEVEDO, 2003). A boa penetração de calda na massa foliar é fundamental para o controle de doenças fúngicas, principalmente, aquelas que se iniciam no terço inferior das plantas, que é o caso da ferrugem da soja. A penetração através da cutícula e subseqüente translocação dentro do tecido da planta é também importante para as substâncias de ação sistêmica (WIRTH et al., 1991).

Os produtos sistêmicos, direcionados às folhas, poderiam ser aplicados com menor densidade de gotas, permitindo o uso de gotas maiores e facilitar a adoção de técnicas como a redução da deriva. Entretanto, no controle da ferrugem, o sucesso sempre tem sido vinculado a maior penetração de gotas na planta, mesmo para fungicidas de ação sistêmica, devendo assim, ser usadas gotas finas ou muito finas (ANTUNIASSI; BAIO, 2004).

Antuniassi (2005) chama a atenção para o controle em aplicações preventivas, as quais têm se mostrado mais eficiente e por esta razão, é grande a demanda por sistemas de aplicação eficiente e de alto rendimento operacional, visando o momento mais adequado. $\mathrm{O}$ uso de diferentes tamanhos de gotas e volumes de calda pode resultar em situações de maior ou menor cobertura das folhas, com potencial influência no desempenho dos fungicidas no controle da doença. O tamanho adequado das gotas possui importância fundamental para a deposição do defensivo sobre o alvo e minimização de perdas ou deriva.

O objetivo da tecnologia de aplicação é colocar a quantidade certa de ingrediente ativo no alvo desejado, com a máxima eficiência, da maneira mais econômica possível e sem afetar o ambiente (DURIGAN, 1989). A tecnologia de aplicação refere-se à qualidade com que se faz o defensivo agrícola atingir o alvo desejado relacionando o tipo de equipamento utilizado, a qualidade de água, o momento da aplicação, as condições ambientais, o tipo de ponta, etc. (SILVA, 2004). 
Os bicos hidráulicos são os principais órgãos do pulverizador e tem por função formar gotas. Na maioria das vezes, a vazão do pulverizador é estabelecida pela vazão do bico; ou da somatória das vazões dos bicos, quando existirem vários. Em alguns casos, a vazão do pulverizador pode ser regulada por dispositivos específicos, assim, os bicos só ficam com a função formadora de gotas (MATUO, 1990). O que se chama genericamente de bico é o conjunto de peças colocadas no final do circuito hidráulico, através do qual a calda é emitida para fora da máquina. Esse conjunto é composto de várias partes, das quais a ponta de pulverização é a mais importante, regulando a vazão, o tamanho das gotas e a forma do jato emitido (CHRISTOFOLETTI, 1999).

Antuniassi (2005) cita que uma característica importante para a definição das estratégias de controle da ferrugem quanto à tecnologia de aplicação é a maneira como os fungicidas sistêmicos se movimentam nas plantas da cultura após a aplicação e absorção. No mercado atual, a maioria dos fungicidas para ferrugem apresenta movimentação no sentido da base para topo de cada folha, com mínima chance de movimentação no sentido contrário e sem a possibilidade de translocação de uma folha para outra.

Para os produtos de contato ou de menor ação sistêmica, o uso de gotas menores e/ou maior volume de calda torna-se necessário, devido a maior dependência com relação à cobertura dos alvos. Os produtos sistêmicos podem ser aplicados com menor densidade de gotas permitindo o uso de gotas maiores, o que facilita a adoção de técnicas para a redução de deriva e melhora a segurança da aplicação aumentando a eficiência. Se usadas de maneira correta, gotas grandes proporcionam bom nível de depósito, mas não proporcionam boas condições de cobertura e penetração (ANTUNIASSI, 2006).

Quando o alvo inclui a parte interna ou inferior das plantas, como no caso de aplicações de fungicidas para ferrugem da soja, é necessária uma boa penetração da nuvem de gotas e neste caso devem ser usadas gotas finas ou muito finas (ANTUNIASSI, 2006).

Wirth et al. (1991) constataram que, para se obter a máxima eficiência nas pulverizações, todas as operações devem ser feitas com a máxima precisão possível. O transporte de ingrediente ativo inicia-se com o preparo da solução, seguido pelo ato da pulverização e continua durante a trajetória e impacto das gotas na superfície da folha. Conforme Antuniassi (2005) e Antuniassi e Baio (2004), esta definição de parâmetros como o tamanho das gotas e volume de aplicação depende diretamente da relação alvo/defensivo e condições climáticas.

O presente trabalho teve como objetivo realizar um diagnóstico das características de desempenho do flutriafol (Impact), comparado a tiofanato metílico + flutriafol (Impact Duo), aplicados com gotas finas e médias, no controle da ferrugem asiática da soja (P. pachyrhizi). 


\section{MATERIAL E MÉTODOS}

O presente trabalho foi conduzido em área comercial da fazenda Ponte de Pedra (grupo Maggi), localizada a $45 \mathrm{~km}$ de Rondonópolis, Estado de Mato Grosso, no período de 18 de fevereiro a 31 de março de 2006. A área está situada a uma altitude de 520 metros, com as seguintes coordenadas geográficas: $16^{\circ} 42^{\prime} 49^{\prime \prime}$ de latitude Sul e 5448' $54^{\prime \prime}$ ' de longitude Oeste. A cultura da soja (cv. Tabarana, estádio R 5.1) ocupava um talhão de 300 ha, semeada no dia 14/11/2005 com $50 \mathrm{~cm}$ de espaçamento no sistema de plantio direto. A colheita foi realizada em 31/03/2006. Antes do ensaio, foram feitas três aplicações de fungicidas para o controle da ferrugem asiática, as quais se encontram descritas na Tabela 1 . O experimento foi instalado por ocasião da quarta aplicação em 18/02/2006.

Tabela 1 - Fungicidas utilizados para o controle da ferrugem asiática ( $P$. pachyrhizi), realizadas antes da instalação do experimento. Rondonópolis/MT, 2006.

\begin{tabular}{|c|c|c|c|c|c|}
\hline \multirow{2}{*}{ Data } & \multirow{2}{*}{ Nome comum } & \multicolumn{2}{|c|}{ Dose por hectare } & \multirow{2}{*}{ Vazão } & \multirow{2}{*}{ Ponta } \\
\hline & & g de i. a. & L de pc & & \\
\hline $4 / 1 / 2006$ & $\begin{array}{c}\text { epoxiconazole }+ \text { piraclostrobin } \\
\text { (Ópera) }\end{array}$ & $66,5+25$ & 0,5 & 30 & CP (aéreo) \\
\hline $22 / 1 / 2006$ & $\begin{array}{c}\text { epoxiconazole }+ \text { piraclostrobin } \\
\text { (Ópera) }\end{array}$ & $66,5+25$ & 0,5 & 85 & $\begin{array}{l}\text { TJ } 11003 \text { (ter- } \\
\text { restre) }\end{array}$ \\
\hline $5 / 2 / 2006$ & $\begin{array}{c}\text { Carbendazin } \\
\text { (Derosal } 500 \text { SC) }\end{array}$ & 250 & 0,5 & 30 & $\begin{array}{l}\text { Cone Teejet } \\
\text { (aéreo) }\end{array}$ \\
\hline
\end{tabular}

g de i. a.: grama de ingrediente ativo; L. de pc: litros de produto comercial.

O ensaio foi realizado durante o ciclo reprodutivo da cultura e dentro do período considerado crítico que a ferrugem asiática (P. pachyrhizi) requer tratamento, para isto foi escolhida uma área uniforme. Para a realização do controle da doença utilizaram-se dois fungicidas: flutriafol 62,5 g i. a./ha (Impact $125 \mathrm{SC}$ ) e tiofanato metílico + flutriafol $300+60$ g i. a. ha ${ }^{-1}$ (Impact DUO), nas doses de 0,5 e $0,6 \mathrm{~L} \mathrm{ha}^{-1}$ do produto comercial, respectivamente. Foram utilizados dois pulverizadores terrestres para otimizar as aplicações, em virtude do uso de dois produtos (uma calda em cada pulverizador, para evitar a troca de calda e interromper a aplicação entre os blocos). Os pulverizadores foram: Pulverizador automotriz 4 x 2 (Uniport), marca Jacto, com tanque de fibra de vidro, capacidade de 2000 litros, barra de 21 metros (Figura 1); Pulverizador automotriz 4 × 2 (Parruda MA 2025 - M), marca Montana, com tanque de fibra de vidro, capacidade de 2200 litros, barra de 25 metros (Figura 2).

Cada pulverizador foi equipado com 43 conjuntos Twin Cap, com duas pontas cada (Figura3), de acordo com os tratamentos propostos. Para a pulverização com gotas finas, utilizou-se o modelo VP ("Va- 
riable Pressure") e para as gotas médias o modelo LD ("Lo-Drift"), ambos da marca Hypro, com perfil de jato leque plano $110^{\circ}, 0,5 \mathrm{~m}$ de espaçamento e com a barra posicionada a $0,5 \mathrm{~m}$ de altura da cultura. No pulverizador Parruda, em função do tamanho maior da barra, os 10 bicos excedentes foram bloqueados para que os dois equipamentos apresentassem a mesma largura de trabalho.

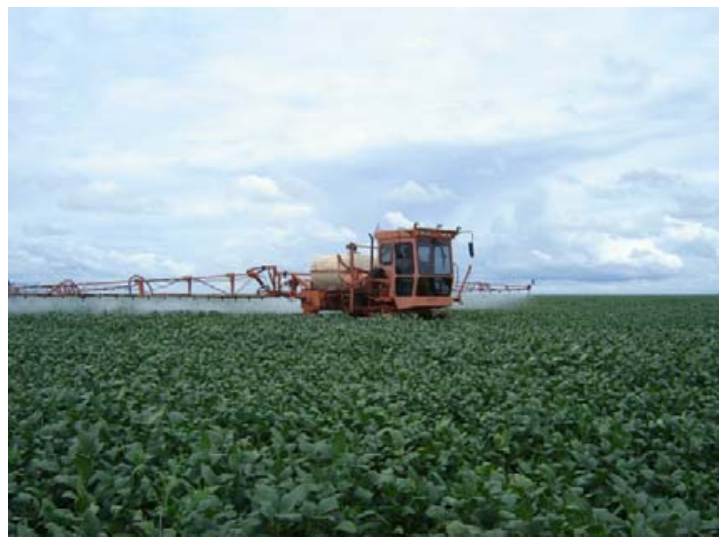

Figura 1 - Uniport Jacto (Foto: Maria A. P. Oliveira).

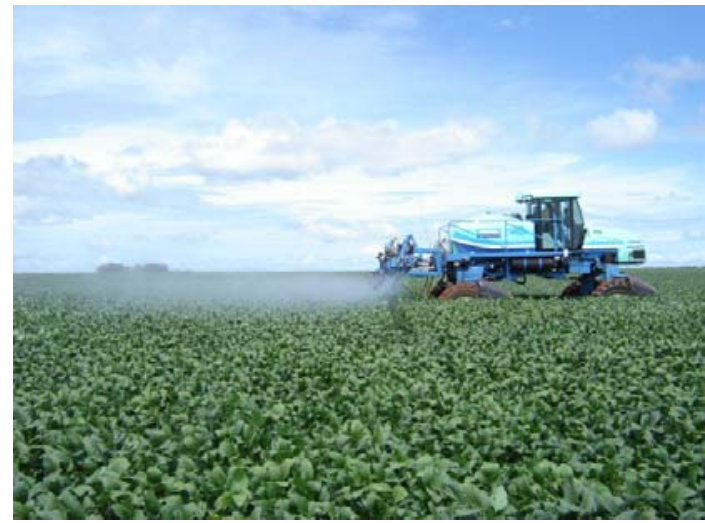

Figura 2 - Parruda Montana (Foto: Maria A. P. Oliveira).
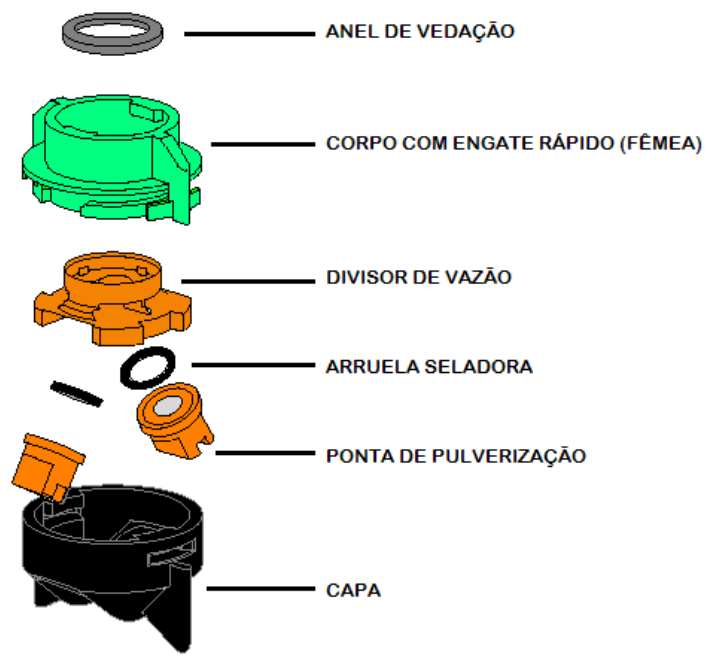

Figura 3 - Componentes do bico (Fonte: Hypro) 
Em todas as aplicações, as condições foram monitoradas de modo que os tratamentos ocorressem de maneira mais uniforme possível. A velocidade durante a aplicação foi de $16 \mathrm{~km} \mathrm{~h}^{-1}$, pressão de 3 bar e volume de calda de $90 \mathrm{~L} \mathrm{ha}^{-1}$. Antes da pulverização os pulverizadores foram calibrados e testados. As aplicações foram em superfície plana, sem obstáculos de maneira que eventuais oscilações de barra não oferecessem diferenças nos tratamentos. A descrição dos tratamentos pode ser visualizada na Tabela 2.

Tabela 2 - Tratamentos, tipo de ponta, classe das gotas (informada pelo fabricante) e fungicidas utilizados no experimento para controle da ferrugem asiática (P. pachyrhizi). Rondonópolis/MT, 2006.

\begin{tabular}{cccc}
\hline Tratamentos & Pontas & $\begin{array}{c}\text { Classe } \\
\text { de gotas } \\
\text { (ASAE S572) }\end{array}$ & Fungicidas \\
\hline $\begin{array}{c}\text { ID GM } \\
\text { Impact Duo Gota Média) } \\
\text { ID GF }\end{array}$ & LD & Médias & tiofanato metílico + flutriafol \\
(Impact Duo Gota Fina) & VP & Finas & tiofanato metílico + flutriafol \\
IP GM & LD & Médias & flutriafol \\
(Impact Gota Média) & & Finas & \\
IP GF & VP & & \\
(Impact Gota Fina) & & &
\end{tabular}

No monitoramento das condições ambientais foram utilizados: termohigrômetro (Lutron HT3004), para verificar a temperatura e umidade relativa do ar e um e anemômetro de hélice (Extech 407445), para medir a velocidade do vento.

O delineamento experimental adotado foi em esquema fatorial $2 \times 2$ com 6 repetições, resultando em 4 tratamentos nas seguintes interações: 2 classes de gotas e 2 fungicidas (Tabela 2). A área experimental constituiu-se de 24 parcelas de aplicação $(50 \mathrm{~m}$ x $21 \mathrm{~m})$. Dentro de cada parcela foi posicionada uma área de avaliação central com 10 × 20 m. Para cada parcela de avaliação havia uma testemunha não tratada fora da área aplicada (com as mesmas dimensões), localizada em direção oposta ao deslocamento do vento no momento da aplicação.

As avaliações da eficiência dos tratamentos no controle da doença foram feitas mediante cálculo da incidência (porcentagem de plantas com ferrugem), severidade (intensidade ou níveis de infecção), desfolha (porcentagem de queda de folhas) e produtividade (pesagem das sementes das parcelas). Para cada uma das análises, as médias dos resultados foram comparadas pelo cálculo do intervalo de confiança 
à $90 \%$. Para a análise da incidência da doença foram consideradas no cálculo folhas que tinham a partir de uma pústula, independente da idade ou condição fisiológica. Na severidade, foi atribuída uma nota de acordo com a escala diagramática de severidade proposta por Godoy et al., (2006), onde as notas estão divididas em seis níveis de porcentagem de infestação. As avaliações da evolução da ferrugem constaram de uma avaliação prévia um dia antes do tratamento (17/02/2006), onde foram analisadas incidência e severidade. Demais avaliações (severidade e desfolha - sendo a desfolha nas duas últimas avaliações) ocorreram em 9, 13, 20 e 27 dias após aplicação (27/02, 03, 10 e 17/03/2006). Com base nos dados de cada repetição e de suas respectivas testemunhas, foram calculados os percentuais de redução da severidade da ferrugem.

$\mathrm{Na}$ desfolha, a análise constou do índice em porcentagem de folhas que caíram quando as testemunhas apresentaram em média $80 \%$ de desfolha. Em cada parcela foram amostradas 50 folhas em 2 alturas: porção superior e inferior da planta, totalizando 100 folhas por parcela. Essa amostragem foi realizada somente nas duas primeiras avaliações. Devido à severidade da doença, houve um aceleramento da desfolha, sendo possível avaliar somente o terço superior das plantas nas demais avaliações.

Após a colheita, foi avaliada a produção através da pesagem das sementes colhidas em duas linhas de cinco metros de cada parcela (valores corrigidos para $13 \%$ de umidade nos grãos). Os valores médios de produtividade nas parcelas tratadas e não tratadas foram usados para o cálculo do valor percentual de ganho em produtividade devido a quarta aplicação de fungicida. A seguir, os dados de produtividade nas 24 testemunhas não tratadas foram submetidos à análise estatística para cálculo dos valores médios, mínimos e máximos de produtividade (intervalo de confiança de 90\%). A produtividade final foi estimada aplicando-se o percentual de ganho do tratamento aos valores médios, mínimos e máximos da área não tratada, obtendo-se assim, valores corrigidos de produtividade para cada tratamento. Este procedimento visou evitar a influência da variabilidade espacial da área, visto que o talhão correspondia a uma área de produção comercial.

\section{RESULTADOS E DISCUSSÃO}

Durante as aplicações as condições climáticas foram as seguintes: umidade relativa entre 87,8 a $78,1 \%$, temperatura entre 23,5 a $28,1{ }^{\circ} \mathrm{C}$ e vento entre 1 a $4,1 \mathrm{~m} / \mathrm{s}$, no período entre $10: 37$ e $12: 31 \mathrm{~h}$.

O presente ensaio foi realizado em condições de controle curativo. Identificou-se através da avaliação da incidência um dia antes da aplicação que a ferrugem estava presente na área com média de $68,6 \%$ de infestação, variando entre 63,6 a 73,7\%, considerando um intervalo de confiança (IC) de $90 \%$, para 48 pontos de amostragem. Nesta mesma análise a severidade média no terço inferior foi de 35,9\% (entre 28,9 e $42,8 \%$ ) e $4,57 \%$ no terço superior (variando entre 2 e $7 \%$ ), considerando IC de $90 \%$. Considerando-se a característica de tratamento curativo tardio, esses valores se assemelham aos encontrados por Camargo et 
al. (2004), onde as plantas estavam com 55\% de área foliar infectada quando os tratamentos foram realizados. Por outro lado, estes valores contrastam com os de Ito et al. (2005), que realizaram as aplicações com apenas $2 \%$ de severidade (entre os estádios R3 e R5), o que pode ser considerado um tratamento curativo inicial.

Forcelini (2003) mostrou que os fungicidas têm sua eficácia muito reduzida quando aplicados após o estabelecimento da ferrugem. Andrade e Andrade (2002) obtiveram resultados que mostraram que no controle químico da ferrugem asiática um atraso de sete dias na aplicação do fungicida (após a detecção da doença), já foi suficiente para o aumento na desfolha em $82 \%$, em relação às parcelas submetidas ao tratamento com fungicida efetuado após o aparecimento da doença. Com atraso de 14 dias, a desfolha aumentou em $155 \%$.

No geral, observou-se que o desenvolvimento precoce da ferrugem, já presente na área antes das aplicações e com grande incidência e severidade, prejudicou a eficiência dos diferentes tratamentos.

A severidade no terço inferior só foi avaliada 9 dias após tratamento (Figura 4). Devido à intensidade da doença houve uma antecipação da desfolha, o que foi ocasionada pela alta pressão de inóculo na área e agressividade do patógeno; uma vez que o fungo se instala primeiro nas partes mais baixas da cultura. Mesmo ocorrendo uma antecipação de desfolha, não houve diferença significativa entre os tratamentos durante esta avaliação.

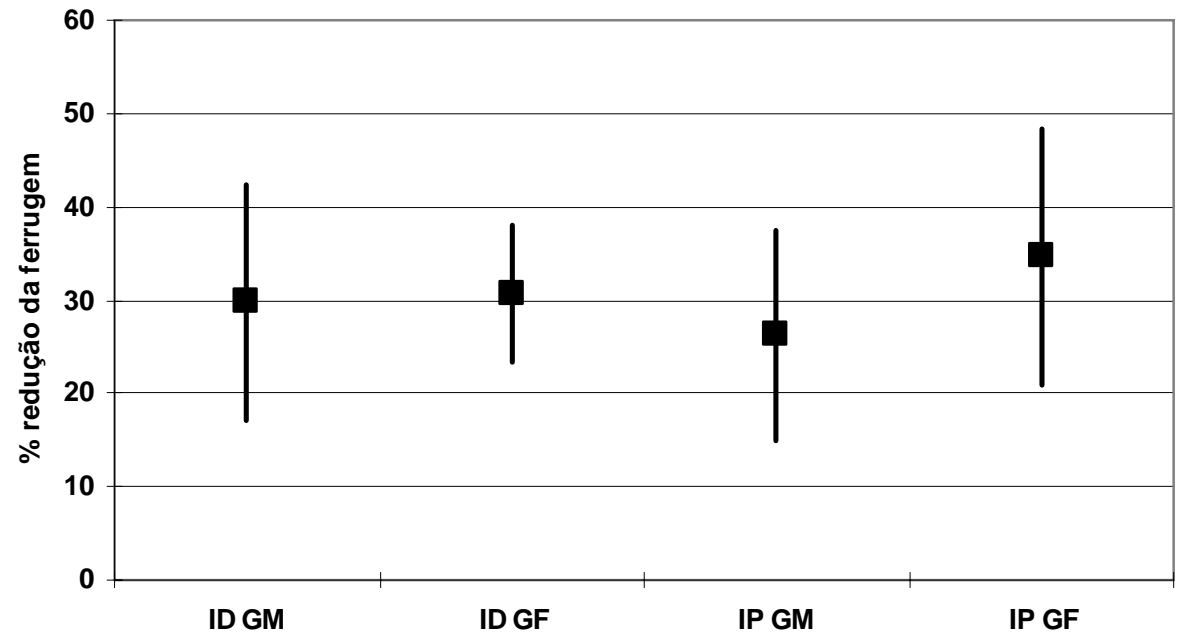

Figura 4 - Porcentagem de redução de ferrugem asiática da soja na comparação de cada tratamento com sua testemunha 9 dias após tratamento (terço inferior das plantas). As barras verticais representam intervalo de confiança (IC $=90 \%, \alpha=0,1)$. Tratamentos: ID GM (Impact Duo com gotas médias), ID GF (Impact Duo com gotas finas), IP GM (Impact com gotas médias), IP GF (Impact com gotas finas). 
Nas avaliações do terço superior em 9, 13, 20 e 27 dias após tratamento (Figuras 5 a 8) os resultados mostraram que não houveram diferenças estatísticas significativas entre os tratamentos. Entretanto, observou-se de maneira geral que em todos os dados houve tendência de melhores resultados para as aplicações com flutriafol, em comparação às aplicações com flutriafol + tiofanato metílico. No que se refere à dose proposta do princípio ativo flutriafol, a diferença entre os dois produtos comerciais existe, porém é pequena $\left(62,5 \mathrm{~g} \mathrm{ha}^{-1}\right.$ para o Impact e $60 \mathrm{~g} \mathrm{ha}^{-1}$ para o Impact Duo). Outros fatores poderiam ser também considerados para referenciar estas tendências, como eventuais diferenças nas formulações ou interação entre os ativos. Estes fatores, entretanto, não foram avaliados neste trabalho e não foram citados diretamente em outros trabalhos com os mesmo ativos, prejudicando a discussão neste sentido.

Ito et al. (2005), utilizando os mesmos princípios ativos e as mesmas doses de produtos do presente trabalho, obtiveram resultados semelhantes entre o controle com flutriafol e tiofanato metílico + flutriafol; diferindo somente no nível de redução da doença. No trabalho citado, entretanto, estes resultados podem ser justificados em função do baixo índice de ferrugem (2\%) que o autor encontrou no início das aplicações. As aplicações com flutriafol apresentaram uma tendência de menor área foliar afetada em R5 $(1,46 \%)$ e diferiu estatisticamente de tiofanato metílico + flutriafol em R6 (12,69\%).

Não foram observadas diferenças marcantes entre as aplicações com gotas finas e médias, contrastando com os resultados obtidos por Antuniassi et al (2004b). Este fato pode ser explicado pelas diferenças de características entre controle preventivo e curativo dos dois trabalhos. Em aplicações preventivas, os melhores resultados têm sido obtidos com o uso de gotas finas ou muito finas (ANTUNIASSI et al., 2004b), principalmente quando se trata de produtos de menor ação sistêmica. Isso se justifica em função da ferrugem inicialmente se instalar nas folhas inferiores da cultura, sendo necessário que a massa de gotas penetre ao máximo na massa de folhas das plantas e proporcione uma maior cobertura, protegendo todas as folhas. Com esse efeito, Antuniassi et al. (2004a) obtiveram resultados de melhor cobertura das folhas em aplicações com gotas muito finas oriundas de pontas do tipo cone, quando comparadas a gotas muito grossas produzidas por pontas de indução de ar. Esta ausência de diferenças entre gotas maiores e menores pode ser explicada também pelas características de ação sistêmica do flutriafol. Este fungicida, por ser um dos mais sistêmicos, apresenta uma redistribuição dentro de cada folha mais efetiva, reduzindo o efeito de melhor cobertura gerado pelas gotas mais finas.

Em condições de aplicações curativas tardias, com alta infestação e infecção (situação do presente trabalho), a quantidade de produto depositado pode se tornar mais importante do que a cobertura, principalmente nas folhas da parte superior das plantas. Estas folhas estão mais sadias do que as inferiores, que geralmente são as primeiras a ficarem totalmente comprometidas, perdendo a importância na manutenção do desenvolvimento da planta. Neste sentido, um exemplo pode ser o trabalho de Antuniassi et al (2005), 
onde tratamentos curativos tardios com gotas muito finas, finas e médias não apresentaram diferenças no controle da ferrugem (terço superior da planta) e na produtividade da cultura.

Do ponto de vista prático, os resultados indicam que a preferência deve ser dada para as gotas médias quando do tratamento visando controle curativo da ferrugem envolvendo os produtos utilizados neste trabalho. Isto se explica pelo fato de que o uso de gotas médias pode facilitar o trabalho dos agricultores no campo, pois as mesmas estendem o período de trabalho na propriedade. Isto ocorre devido às limitações de uso das gotas finas e muito finas, principalmente em função dos problemas relacionados à deriva e a evaporação, onde as gotas médias representam uma escolha de menor risco.

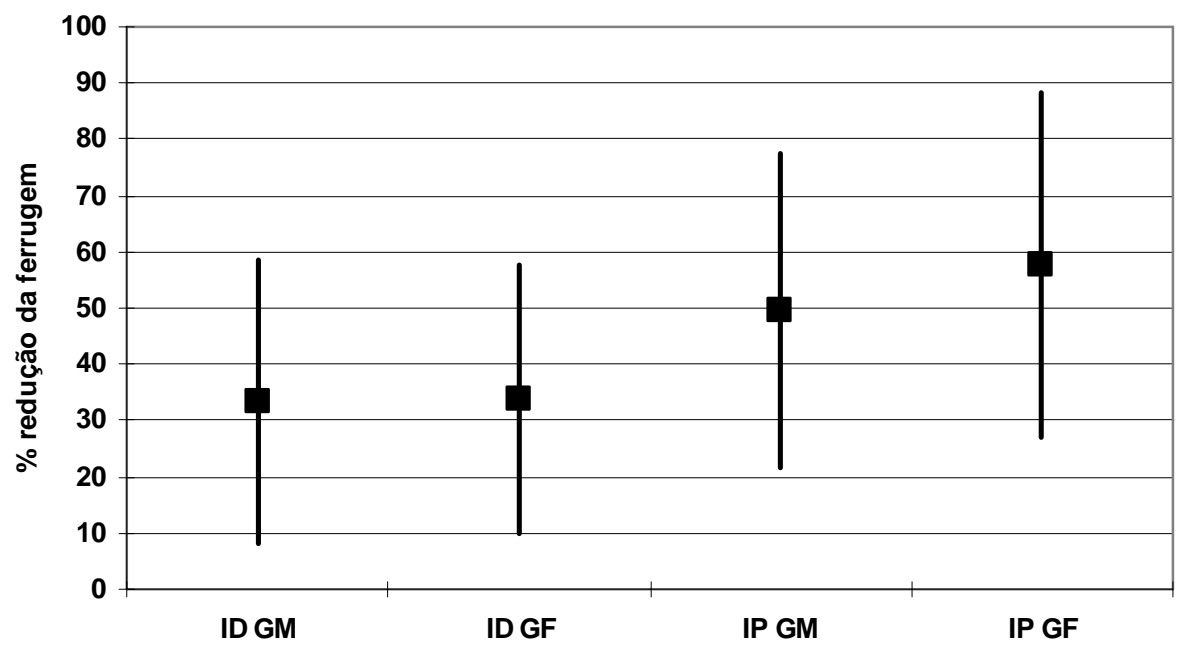

Figura 5 - Porcentagem de redução de ferrugem asiática da soja na comparação de cada tratamento com a sua testemunha 9 dias após tratamento (posição superior das plantas). As barras verticais representam intervalo de confiança (IC $=90 \%, \alpha=0,1$ ). Tratamentos: ID GM (Impact Duo com gotas médias), ID GF (Impact Duo com gotas finas), IP GM (Impact com gotas médias), IP GF (Impact com gotas finas). 


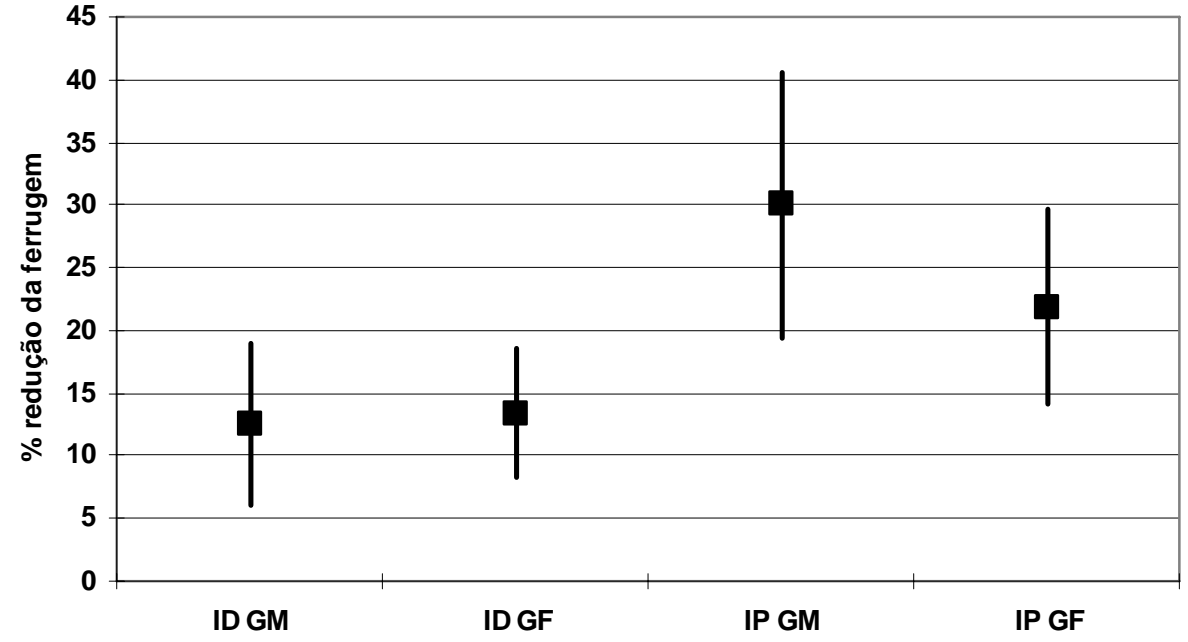

Figura 6 - Porcentagem de redução de ferrugem asiática da soja na comparação de cada tratamento com a sua testemunha 13 dias após tratamento (posição superior das plantas). As barras verticais representam intervalo de confiança (IC $=90 \%, \alpha=0,1)$. Tratamentos: ID GM (Impact Duo com gotas médias), ID GF (Impact Duo com gotas finas), IP GM (Impact com gotas médias), IP GF (Impact com gotas finas).

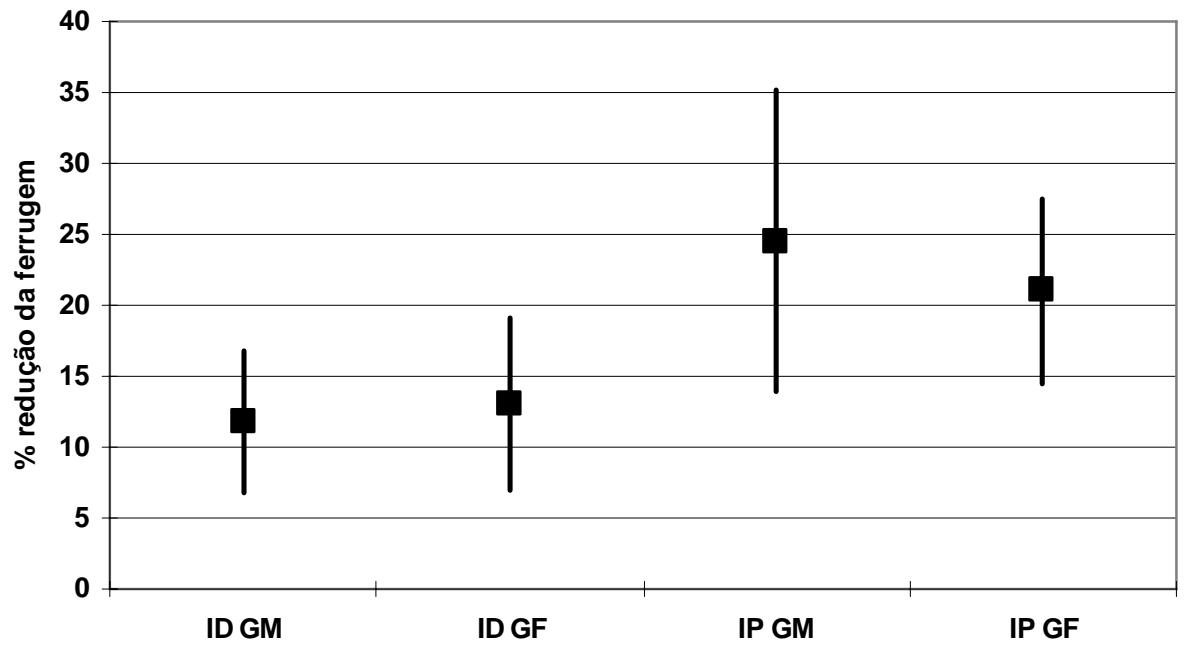

Figura 7 - Porcentagem de redução de ferrugem asiática da soja na comparação de cada tratamento com a sua testemunha 20 dias após tratamento (posição superior das plantas). As barras verticais representam intervalo de confiança (IC $=90 \%, \alpha=0,1)$. Tratamentos: ID GM (Impact Duo com gotas médias), ID GF (Impact Duo com gotas finas), IP GM (Impact com gotas médias), IP GF (Impact com gotas finas). 


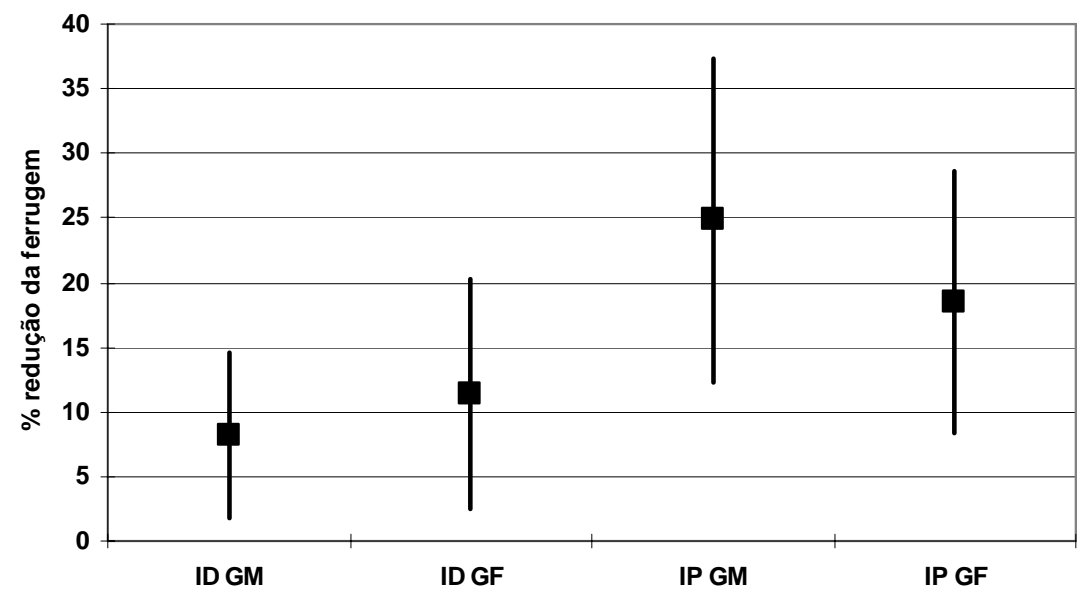

Figura 8 - Porcentagem de redução de ferrugem asiática da soja na comparação de cada tratamento com a sua testemunha 27 dias após tratamento (posição superior das plantas). As barras verticais representam intervalo de confiança (IC $=90 \%, \alpha=0,1)$. Tratamentos: ID GM (Impact Duo com gotas médias), ID GF (Impact Duo com gotas finas), IP GM (Impact com gotas médias), IP GF (Impact com gotas finas).

A severidade observada durante as avaliações foi refletida no índice de desfolha, pois, aos 20 dias após tratamento, a porcentagem média variou conforme os tratamentos aplicados. As parcelas tratadas com flutriafol (tratamentos IP GM e IP GF) obtiveram um índice menor de desfolha, como ocorreu também aos 27 dias após o tratamento (Figuras 9 e 10). Neste sentido, quanto mais cedo ocorrer a desfolha, menor será o tamanho dos grãos de soja e maior será as perdas no rendimento da cultura (YORINORI et al., 2004).

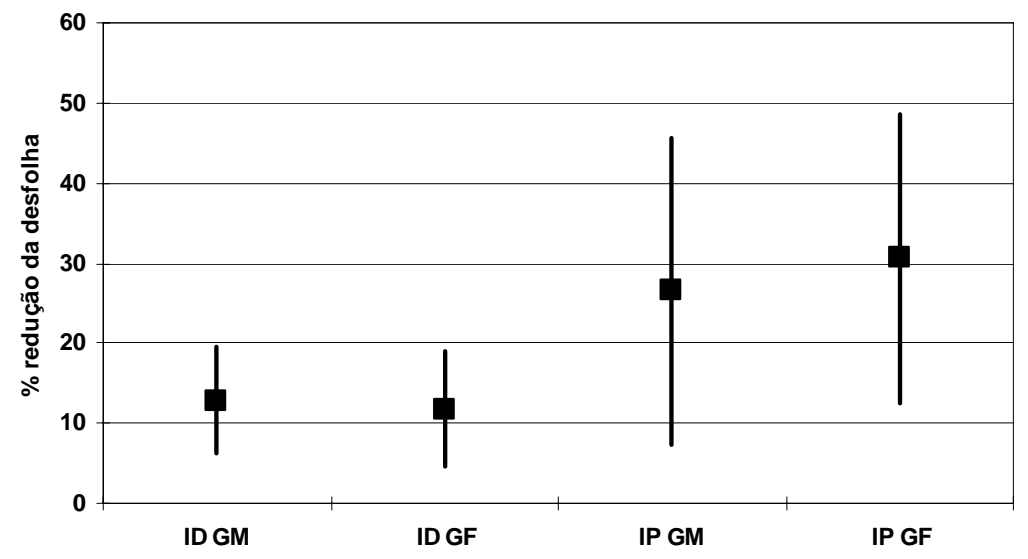

Figura 9 - Porcentagem de redução de desfolha na comparação de cada tratamento com a sua testemunha 20 dias após tratamento (posição superior das plantas). As barras verticais representam intervalo de confi- 
ança (IC $=90 \%, \alpha=0,1)$. Tratamentos: ID GM (Impact Duo com gotas médias), ID GF (Impact Duo com gotas finas), IP GM (Impact com gotas médias), IP GF (Impact com gotas finas).

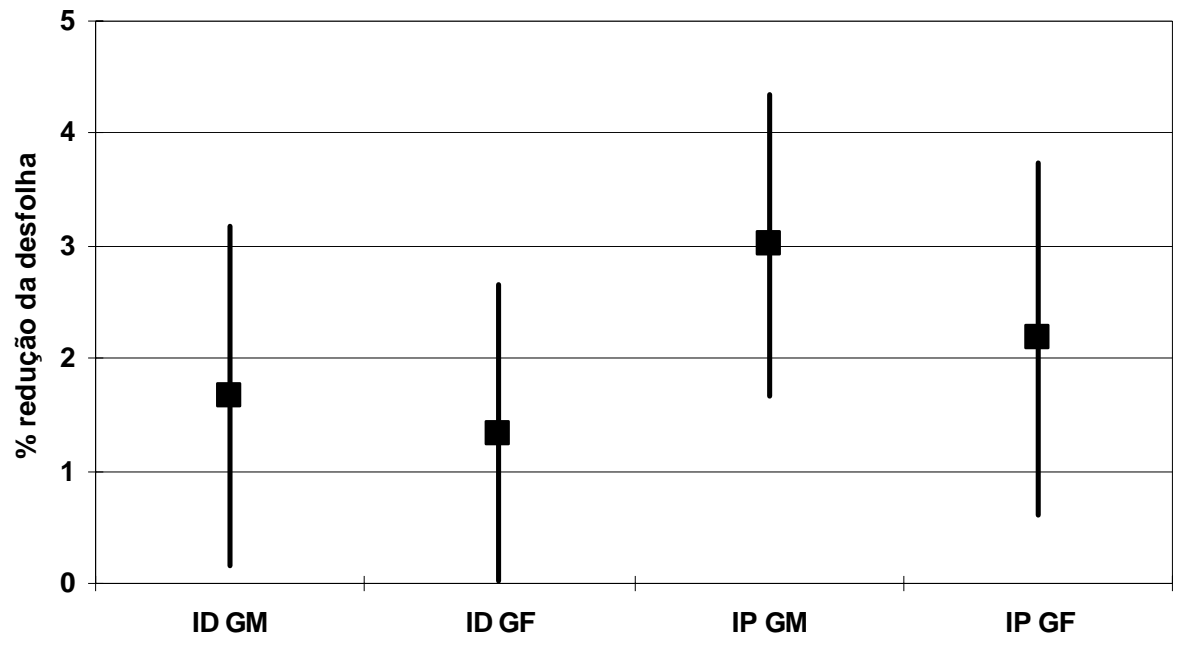

Figura 10 - Porcentagem de redução de desfolha na comparação de cada tratamento com a sua testemunha 27 dias após tratamento (posição superior das plantas). Barras verticais representam intervalo de confiança (IC $=90 \%, \alpha=0,1)$. Tratamentos: ID GM (Impact Duo com gotas médias), ID GF (Impact Duo com gotas finas), IP GM (Impact com gotas médias), IP GF (Impact com gotas finas).

A tendência de menor produtividade nos tratamento ID GM e ID GF (Figura 11) pode estar relacionada também à antecipação da desfolha que a doença causou nas plantas. A desfolha teve reflexos negativos na massa de sementes e, consequentemente, afetou a produtividade. Entretanto, é importante ressaltar que as diferenças observadas não foram significativas considerando-se o IC de 90\%.

Os resultados de produtividade do presente trabalho se assemelham aos encontrados por Ito et al. (2005), que obtiveram um maior peso de 100 grãos com flutriafol a $0,5 \mathrm{~L} \mathrm{ha}^{-1}$, diferindo estatisticamente de flutriafol + tiofanato metílico a $0,6 \mathrm{~L} \mathrm{ha}^{-1}$. 


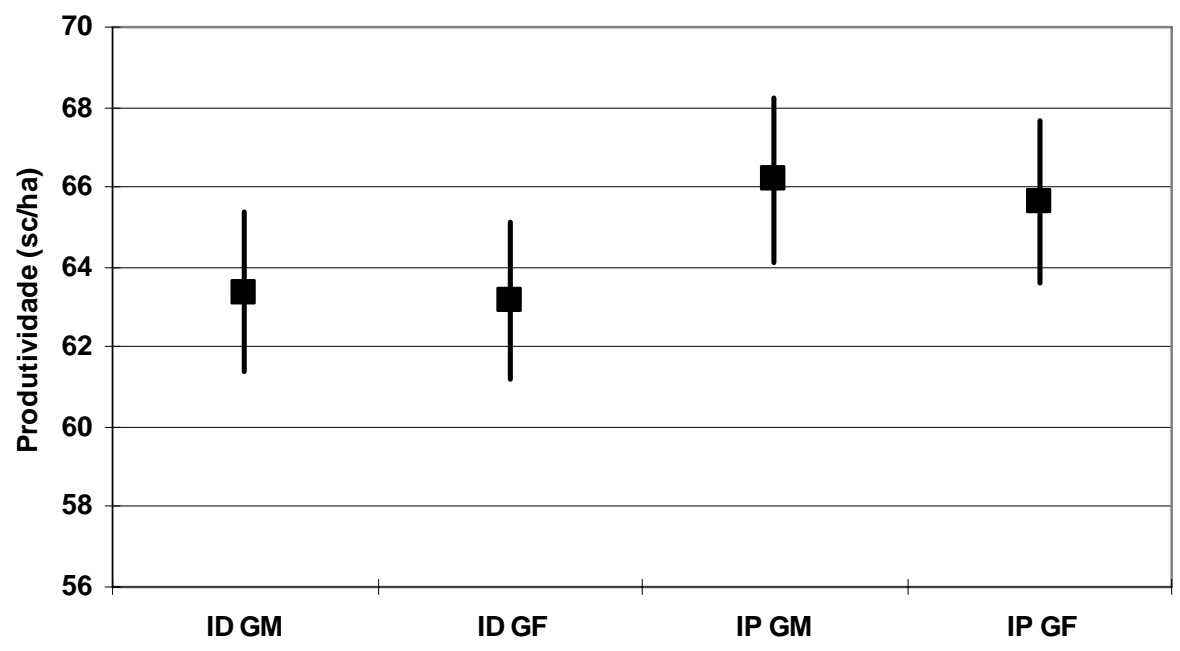

Figura 11 - Produtividade em sacas por hectare. As barras verticais representam intervalo de confiança (IC $=90 \%, \alpha=0,1)$. Tratamentos: ID GM (Impact Duo com gotas médias), ID GF (Impact Duo com gotas finas), IP GM (Impact com gotas médias), IP GF (Impact com gotas finas).

\section{CONCLUSÕES}

De acordo com as condições em que este estudo foi desenvolvido e na análise dos resultados obtidos, pode-se concluir que os fungicidas utilizados (flutriafol e a mistura flutriafol + tiofanato metílico) foram eficazes no controle curativo da ferrugem asiática da soja; não havendo diferença entre os mesmos nas análises de severidade, desfolha e produtividade. Não houveram diferenças significativas quanto ao uso de gotas finas e médias em todas as análises realizadas.

\section{REFERÊNCIAS}

ANDRADE, P. J. M.; ANDRADE, D. F. A. A. Ferrugem asiática: uma ameaça à sojicultura brasileira. Dourados: EMBRAPA Agropecuária Oeste, 2002. 11 p. (Série Circular Técnica, 11).

ANTUNIASSI, U. R. Aplicação aérea no controle da ferrugem da soja. In: RAETANO, C. G.; ANTUNIASSI, U. R. (eds.). Qualidade em tecnologia de aplicação. Botucatu: FEPAF, 2004. p. 1-7. 
ANTUNIASSI, U. R. et al. Avaliação da cobertura de folhas em aplicações terrestres com diferentes tipos de pontas. In: SIMPÓSIO INTERNACIONAL DE TECNOLOGIA DE APLICAÇÃO DE AGROTÓXICOS, 3., 2004, Botucatu. Anais... Botucatu: FEPAF, 2004a. p. 48-51.

. Controle de ferrugem da soja através de aplicações aéreas e terrestres. In: SIMPÓSIO INTERNACIONAL DE TECNOLOGIA DE APLICAÇÃO DE AGROTÓXICOS, 3., 2004, Botucatu. Anais... Botucatu: FEPAF, 2004b. p. 92-95.

ANTUNIASSI, U. R. et al. Desempenho de sistema de aplicação terrestre para controle da ferrugem de soja. In: REUNIÃO DE PESQUISA DE SOJA DA REGIÃO CENTRAL DO BRASIL, 27., 2005, Cornélio Procópio. Resumos... Londrina: EMBRAPA SOJA, 2005. p. 217-218.

ANTUNIASSI, U. R. Tecnologia de aplicação de defensivos na cultura da soja. In: SUZUKI, S., YUYAMA, M. M., CAMACHO, S. A. Boletim Técnico de Pesquisa Soja 2006. Rondonópolis, MT: Fundação Mato Grosso, 2006.

ANTUNIASSI, U. R. Tecnologia de aplicação para o controle da ferrugem da soja. In: WORKSHOP BRASILEIRO SOBRE A FERRUGEM ASIÁTICA, 1., 2005, Uberlândia. Coletânia... Uberlândia: EDUFU, 2005. p. 193-219.

ANTUNIASSI, U. R; BAIO, F. H. R. Tecnologia de aplicação de defensivos. In: VARGAS, L.; ROMAN, E. S. (eds). Manual de manejo e controle de plantas daninhas. Bento Gonçalves: EMBRAPA Uva e Vinho, 2004. p. 145-184.

AZEVEDO, L. A. S. Fungicidas protetores: fundamentos para o uso racional. São Paulo, 2003. 319 p.

BEDENDO, I. P. Ferrugens. In: BERGAMIN FILHO, A.; KIMATI, H.; AMORIM, L. (eds.). Manual de fitopatologia. 3. ed. São Paulo: Agronômica Ceres, 1995. p. 872-880.

BROMFIELD, K. R. Soybean rust. St. Paul: APS Press, 1984. 65 p. (Monography 11).

CAMARGO et al. Controle da ferrugem da soja (Phakopsora pachyrhizi) em aplicações tratorizadas com bicos hidráulico e centrífugo (Baixo Volume Oleoso). In: SIMPÓSIO INTERNACIONAL DE TECNO- 
LOGIA DE APLICAÇÃO DE AGROTÓXICOS, 3., 2004, Botucatu. Anais... Botucatu: FEPAF, 2004a. p. 88-91.

CAMARGO, T. V. Aplicação aérea e terrestre de fungicidas no controle da ferrugem da soja. In: WORKSHOP BRASILEIRO SOBRE A FERRUGEM ASIÁTICA, 1., 2005, Uberlândia. Coletânia... Uberlândia: EDUFU, 2005. p. 181-191.

CHRISTOFOLETTI, J. C. Considerações sobre a deriva nas pulverizações agrícolas e seu controle. São Paulo: Teejet South América, 1999. 15 p.

DURIGAN, J. C. Comportamento de herbicidas no ambiente. In: SEMINÁRIO TÉCNICO SOBRE PLANTAS DANINHAS E O USO DE HERBICIDAS EM REFLORESTAMENTO, 1989, Rio de Janeiro. Anais... Rio de Janeiro: SBS/ABRACAV/SIF, 1989. (paginação irregular).

EMPRESA BRASILEIRA DE PESQUISA AGROPEUÁRIA. Tecnologias de produção de soja - Região Central do Brasil - 2005. Londrina: EMBRAPA Soja; EMBRAPA Cerrados; EMBRAPA Agropecuária Oeste; Fundação Meridional, 2004. 239 p.

FORCELINI, C. A. A ferrugem pode ser manejada. Atualidades Agrícolas, v. 3, p. 8-11, 2003.

GODOY, C. V.; KOGA, L. J.; CANTERI, M. G. Diagramatic scale for assessment of soybean rust severity. Fitopatologia Brasileira, Brasília, v. 31, n. 1, p. 63-68, 2006.

ITO, M. F. et al. Controle da ferrugem asiática da soja com o fungicida Celeiro/Impact Duo. In: REUNIÃO DE PESQUISA DE SOJA DA REGIÃO CENTRAL DO BRASIL, 27., 2005, Cornélio Procópio. Resumos... Londrina: EMBRAPA SOJA, 2005. p. 197-198.

MATUO, T. Técnicas de aplicação de defensives agrícolas. Jaboticabal. FUNEP, 1990. 139p.

MELO FILHO, G. A.; RICHETTI, A. Custo do controle químico da ferrugem asiática da soja. 2. ed. Dourados: EMBRAPA Agropecuária Oeste, 2004. (Comunicado Técnico, n. 93).

NUNES JÚNIOR, J. Evolução e perspectivas para a próxima safra. Revista Plantio Direto, Passo Fundo, p. 28-31, nov/dez. 2004. 
ONO, Y.; BURITICA, P.; HENNEN, J.F. Delimitation of Phakopsora, Physopella and Cerotelium and their species on Leguminosae. Mycological Research, West Lafayette, n. 96, p. 825-850, 1992.

SILVA, L. H. C. P. Ferrugem se instala em Rio Verde. Força ruralista. Rio Verde, p. 5, jan. 2004.

WIRTH, W., STORP, S., JACOBSEN, W. Mechanisms controlling leaf retention of agricultural spray solutions. Pesticy Science., Kyoto, v. 33, p 411-20, 1991.

YORINORI, J. T; JÚNIOR, J. N.; LAZZAROTTO, J. J. Ferrugem “asiática” da soja no Brasil: evolução, importância econômica e controle. Londrina: EMBRAPA Soja, 2004. 36 p. (documentos, 247).

YORINORI, J. T; PAIVA, W. M. Ferrugem da soja Phakopsora pachyrhizi. Londrina: EMBRAPA CNPSo, 2002. 8 Folder.

YORINORI, J.T. Situação atual das doenças potenciais no cone sul. In: CONGRESSO BRASILEIRO DE SOJA, 2., 2002, Foz do Iguaçu. Resumos... Foz do Iguaçu: EMBRAPA, 2002. p.171- 186. 